\title{
Comportamiento de las fábricas no estructurales en el terremoto de Lorca
}

\author{
Lutz Hermanns, Alberto Fraile de LERMA, ENRIQue Alarcón Álvarez \& \\ RAMÓN ÁLVAREZ CABAL
}

Escuela Técnica Superior de Ingenieros Industriales

Universidad Politécnica de Madrid

lhermanns@etsii.upm.es

Recibido: $25 / 10 / 2012$

Aceptado: 17/09/2012

\section{Resumen}

En lo que sigue se describen los efectos del terremoto del 11 de mayo de 2011 en las fachadas y particiones de los edificios de Lorca y se analiza la interacción entre estos elementos, tradicionalmente considerados como no-estructurales, y la propia estructura. Las observaciones de campo se justifican a partir de los resultados de algunos modelos numéricos simples y de todo ello se concluye la importancia fundamental de las fábricas en el comportamiento de los edificios durante el terremoto

Palabras clave: Terremoto de Lorca, ingeniería sísmica, fabricas no estructurales.

\begin{abstract}
Behavior of non-structural masonry in the Lorca earthquake Abstract

Lorca 11 May 2011 earthquake effects are described on both facades and partitions of Lorca buildings and the relationship among these elements, traditionally regarded as non-structural, and the structure itself is analysed. In addition, field observations are reproduced with simple numerical models. From this study we prove the high importance of the masonry in the building behaviour during this earthquake.
\end{abstract}

Keywords: Lorca earthquake, earthquake engineering, non-structural masonry.

\section{Referencia normalizada}

Hermanns, L., Fraile, A., Alarcón, E., Álvarez, R. (2012). Comportamiento de las fábricas no estructurales en el terremoto de Lorca. Física de la Tierra, Vol. 24, 315-341.

Sumario: Introducción. 1. Las fábricas como elementos pasivos. 2. Las fábricas como elementos activos. 3. Plantas blandas. 4. Daños en pilares. 5. Formas de fallo. 5.1. Deslizamiento de la junta constructiva pilar-viga. 5.2. Fallo del panel en tendales. 5.3. Fallo conjunto panel-pilar. 6. Solicitaciones sobre los pilares. 7. Resistencia de los pilares. 8. Discusión de resultados. Referencias bibliográficas. 


\section{Introducción}

El riesgo sísmico de la ciudad de Lorca puede considerarse entre bajo y medio, según se deduce de los datos históricos de la actividad sísmica de la región. El histograma de la

Figura 1 representa el número de ocurrencias frente a la intensidad en la región para intensidades de valor IV y superiores.

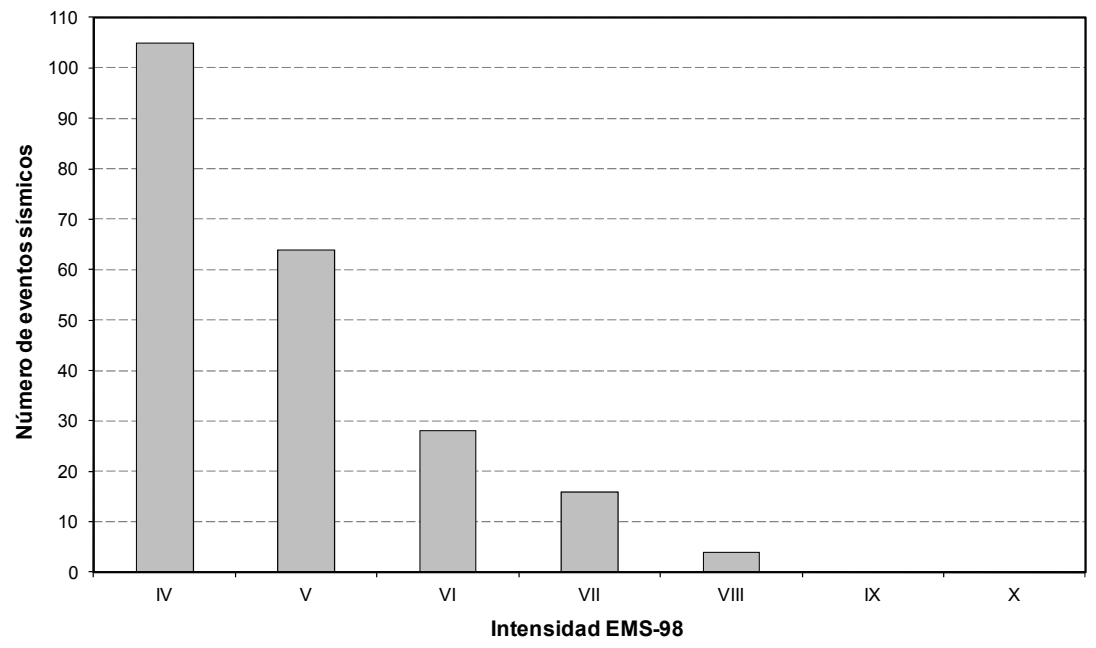

Figura 1. Región definida entre latitud norte $37,2^{\circ}$ y $38,2^{\circ}$ y longitud oeste $-1,1^{\circ}$ y $-2,2^{\circ}$. (IGN)

De acuerdo con la norma de construcción sísmica española (NCSE, 2002) el valor de la aceleración básica $a_{b}$ considerando un periodo de retorno de 500 años es del $12 \% \mathrm{~g}$. Este valor de PGA no corresponde al sismo en roca sino en un suelo duro (tipo B del EN-1998, 2004).

El terremoto de Lorca del 11 de mayo de 2011 fue registrado por la red sísmica nacional, alcanzándose valores, en un acelerógrafo situado a $3 \mathrm{~km}$ del epicentro, del $36 \% \mathrm{~g}$ en dirección $\mathrm{N}-\mathrm{S}, 15 \% \mathrm{~g}$ en dirección E-O y el $12 \% \mathrm{~g}$ en dirección vertical, claramente superiores al valor de proyecto (Cabañas et al. 2011). En la figura 2 se muestran las historias de aceleración registradas por el acelerógrafo y los espectros de respuesta elástica obtenidos con un 5\% de amortiguamiento.

La imagen de la ciudad de Lorca inmediatamente tras el terremoto de mayo de 2011 no era la de los edificios colapsados que cabría esperar de una solicitación que excedía ampliamente las previsiones normativas. De hecho, durante el terremoto únicamente se produjo el colapso de un edificio, un bloque de viviendas de construcción reciente. 

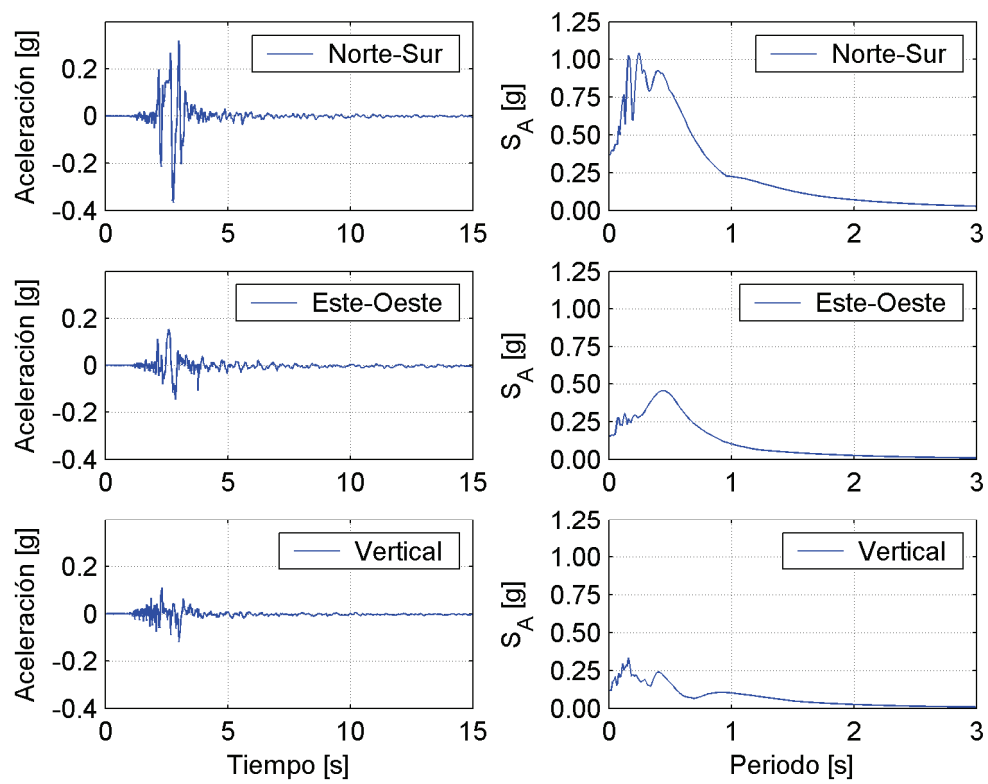

Figura 2

No obstante, las calles de la ciudad presentaban tras el terremoto el aspecto que intenta reflejar la Figura 3. En las aceras, sobre los automóviles y dispersos en la calzada aparecían restos de albañilería, en ocasiones paños enteros de fachadas, parapetos y todo tipo de cascotes. De hecho, fue precisamente la caída de estos elementos la que provocó la mayoría de los daños a personas.

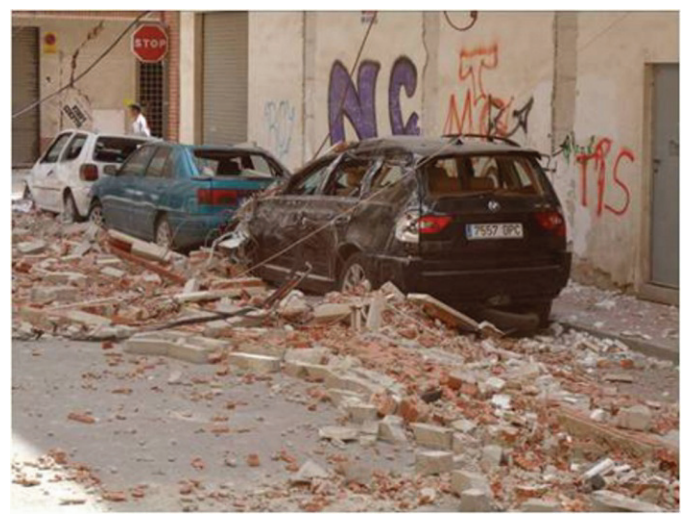

Figura 3 
La caída de las fábricas parecía responder a dos mecanismos diferentes:

1. Los parapetos, chimeneas, y en menor medida, algunos paños de fachada de las plantas más altas parecían haber fallado a flexión ante fuerzas normales a su plano. Como la capacidad de las fábricas ante acciones de flexión no es comparable a la de la estructura no se produce interacción alguna con ésta. Las fábricas se comportan en este sentido como elementos pasivos sujetos a la acción del terremoto amplificada en función de su posición en la estructura. Entre otros aspectos se trata en la guía práctica (FEMA, 2011). (Figura 4)

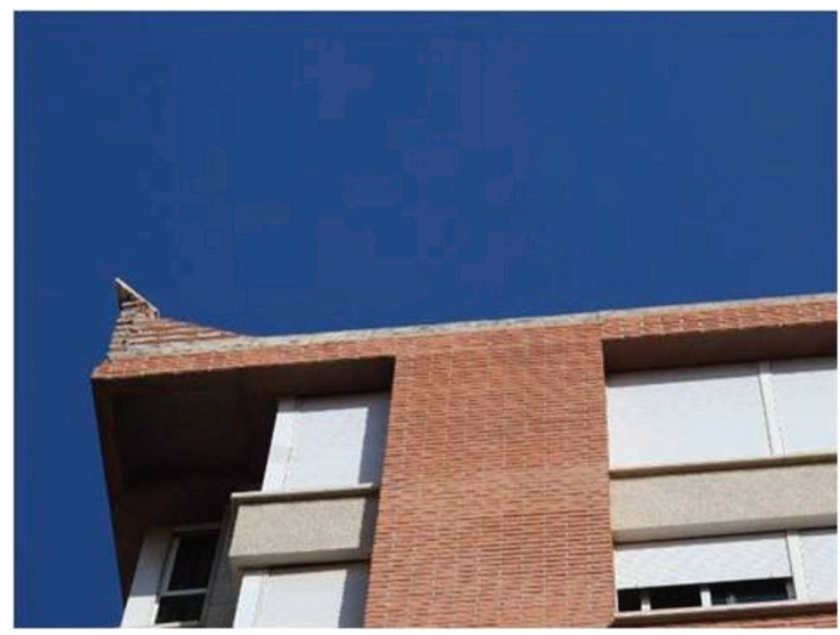

Figura 4

2. En el extremo opuesto, muchos paneles de fábrica en las plantas más bajas parecían haber colapsado ante el efecto de las acciones en su plano, desplazamientos de la estructura que los confina, y en algunos casos, impactos entre edificios separados por juntas de insuficiente abertura. En este caso el cálculo más elemental permite demostrar que la rigidez de la fábrica es comparable a la de la estructura, cuya respuesta condiciona. En este sentido las fábricas constituyen elementos activos que interaccionan con la estructura. (Figura 5)

Aunque en algunos casos se superponían las dos formas de fallo, con cierta generalidad los petos y cerramientos de las plantas altas fallaban conforme al primero de los esquemas descritos y los paneles de las plantas bajas lo hacían conforme al segundo.

En el capítulo 2 del artículo se incide en el comportamiento de las fábricas como elementos pasivos, mientras que en el capítulo 3 y siguientes se profundiza 
en su comportamiento como elementos activos. Así, en el capítulo 3 se realiza un catálogo de las posibles causas de fallo de la estructura inducidos por la presencia de la fábrica. De ellos se han escogido los dos que se han considerado más significativos: los efectos de planta blanda y los daños en pilares que se desarrollan en los capítulos 4 y 5 .

La última parte del artículo se centra en el estudio local de la interacción entre pilares y fábrica identificando, en el capítulo 6, las formas de fallo observadas. Con objeto de clarificar los motivos en el capítulo 7 se muestran los resultados del cálculo mediante elementos finitos de dos estructuras de edificios en las que se incluye la fábrica en algunos paños para evaluar el efecto de la interacción, obteniendo valores de los esfuerzos que deben soportar los pilares. En el capítulo 8 se describe la resistencia de la sección del pilar ante los modos de fallo identificados. Finalmente, en el capítulo 9 se discuten los resultados obtenidos que justifican el daño en los pilares.

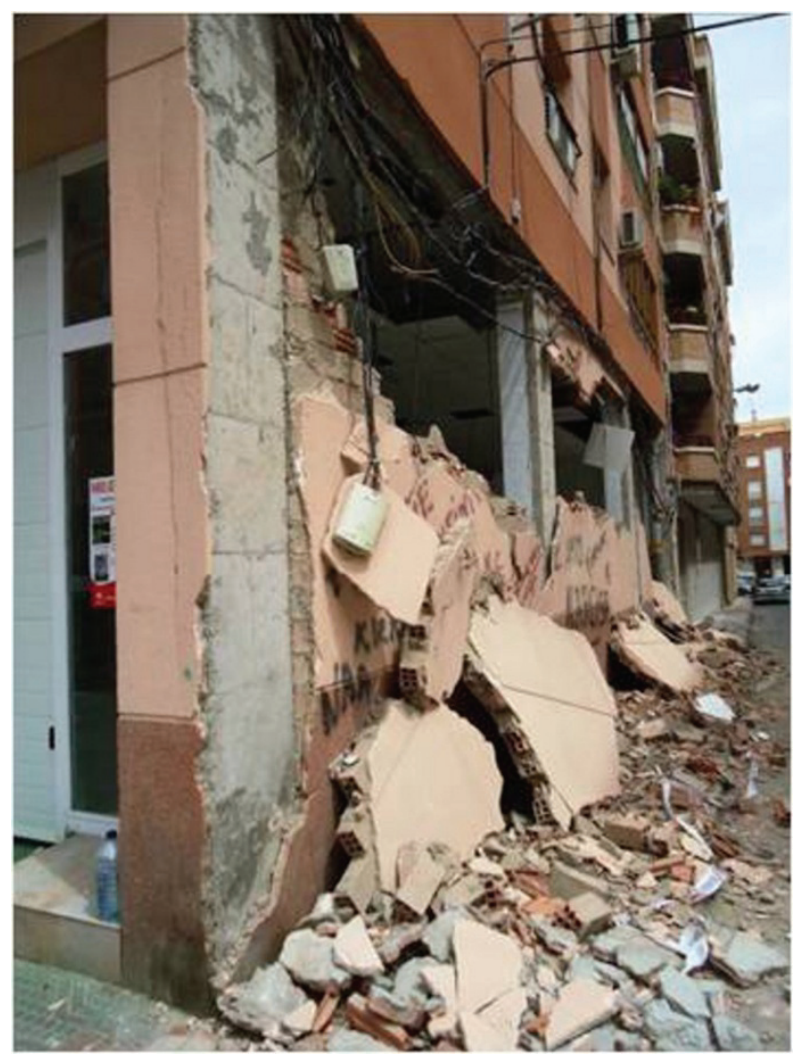

Figura 5 


\section{Las fábricas como elementos pasivos}

Las acciones normales al plano de las fábricas son las correspondientes a las fuerzas de inercia derivadas de la acción sísmica, fuerzas amplificadas por el propio edificio.

La figura 6a muestra la comparación entre la aceleración horizontal del suelo (componente $\mathrm{N}-\mathrm{S}$ del registro de aceleración) y la que se produciría en la azotea de un edificio típico de la ciudad, un edificio de ocho plantas y período en torno a 0,72 segundos (valor ajustado a la expresión habitual $0.09 \cdot n$, siendo " $n$ " número de plantas). Se observan valores semejantes a los de la gravedad, que equivalen al peso propio del parapeto en la hipótesis de que éste se construyera en voladizo horizontal. Naturalmente, la resistencia de las fábricas en la sección en la que apoyan sobre los forjados de cubierta es muy inferior a la solicitación inducida por un parapeto de las dimensiones habituales (altura en torno a 1,2 $\mathrm{m}$ y espesor de $12 \mathrm{~cm})$.

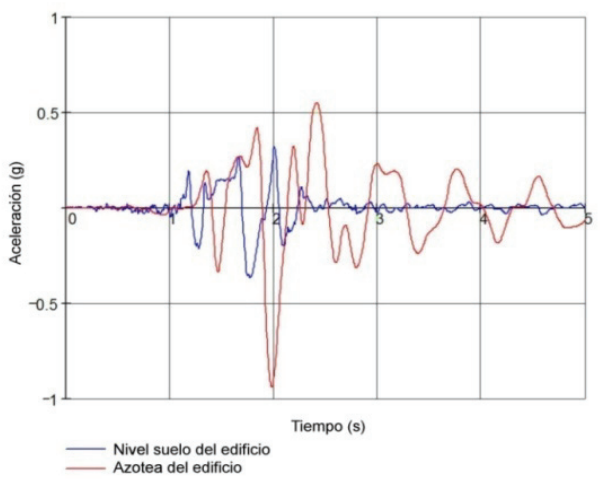

a)

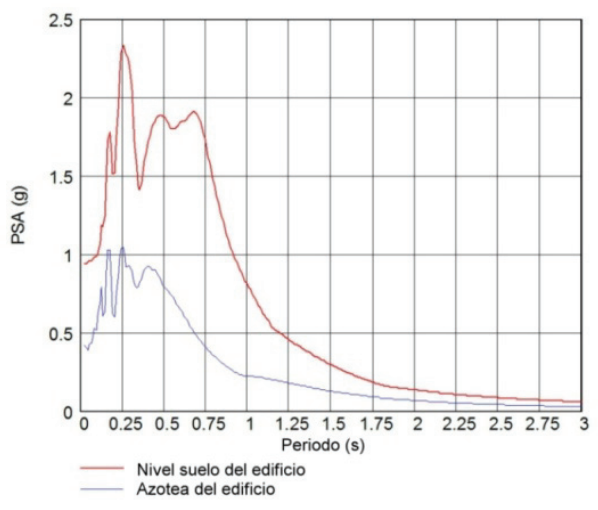

b)

Figura 6

Como generalización elemental del planteamiento descrito se dibujan (Figura 6b) los espectros correspondientes al registro del sismo en superficie y al integrado a la altura de la azotea del edificio tipo descrito en el párrafo anterior. Se observa la importancia de la amplificación inducida por el propio edificio, amplificación que justifica la gran cantidad de daños en los elementos situados en las cubiertas (Figura 7). 


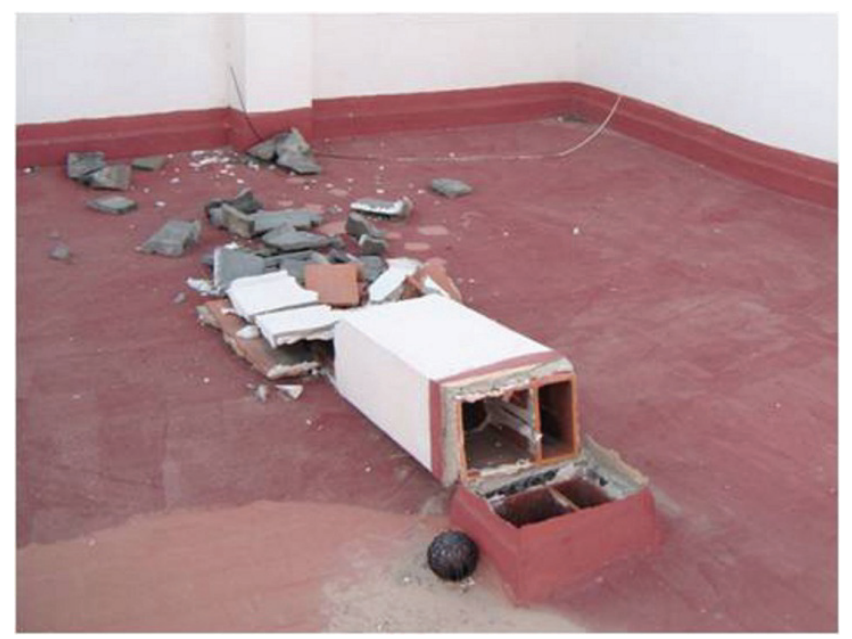

Figura 7

\section{Las fábricas como elementos activos}

Un recorrido por la ciudad de Lorca tras el terremoto permitía catalogar muchos de los daños conforme a los más habituales criterios de fallo por interacción de la estructura con los cerramientos y particiones.

Dentro de este tipo de problemas se puede distinguir entre los relativos a la configuración inicial de las fábricas y los que se presentan conforme la progresiva rotura de los paños más solicitados modifica la distribución de rigideces y la trayectoria de los esfuerzos horizontales. En concreto, y dentro del primer grupo, eran patentes los siguientes efectos:

1. Rigidización global del edificio. Ello resultaba especialmente patente en edificios que no disponían de un esquema resistente eficaz ante acciones horizontales y que, sin embargo, resistieron la acción sísmica sin mostrar una distribución de daños muy diferente a la que presentaban edificios que sí disponían de tal esquema resistente capaz (aunque, en la mayoría de los casos, flexible en exceso).

2. Torsiones inducidas por la asimetría que los paños de cerramiento introducen en la distribución en planta de las rigideces. El ejemplo más señalado se refiere a los edificios que forman esquina en las manzanas de urbanización. Algunos de los edificios con mayores daños (y el único colapsado) se encontraban en esta situación. La gran rigidez de los cerramientos de medianería limita el desplazamiento de la alineación correspondiente frente al resto de las alineaciones, produciendo las consiguientes torsiones.

3. Efectos de "planta blanda" causados por la diferencia entre los cerramientos comerciales de las plantas bajas, normalmente más ligeros, y los de 
las plantas destinadas a viviendas, formados por paneles de fábrica de gran rigidez (Figura 8a).

4. Existencia de "pilares cautivos" (Figura 8b), pilares cuyo desplazamiento horizontal queda coartado parcialmente por los cerramientos, reduciendo su altura libre.

5. Concentración de los esfuerzos en los pilares que encierran recuadros aislados de fábrica (Figura 9), concentración relativa a la distribución de cortantes obtenida en los cálculos de proyecto, en los que habitualmente no se considera la existencia de estos rellenos. Se trata de una situación frecuente en cierto tipo de edificios residenciales, en los que la planta baja no presenta más cerramientos que los correspondientes a los núcleos de escaleras y ascensores, limitándose por tanto a uno o dos vanos en cada dirección.

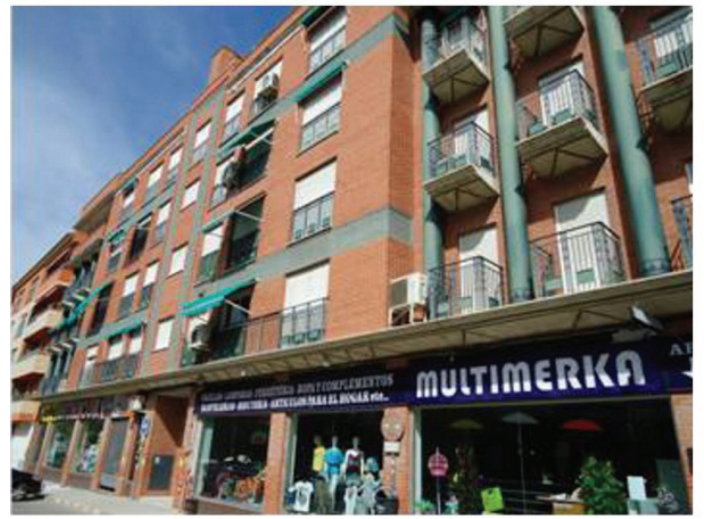

a)

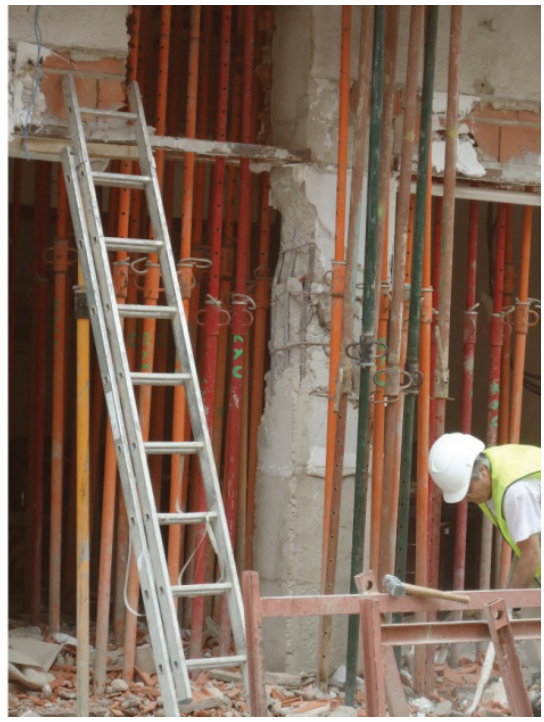

b)

Figura 8 


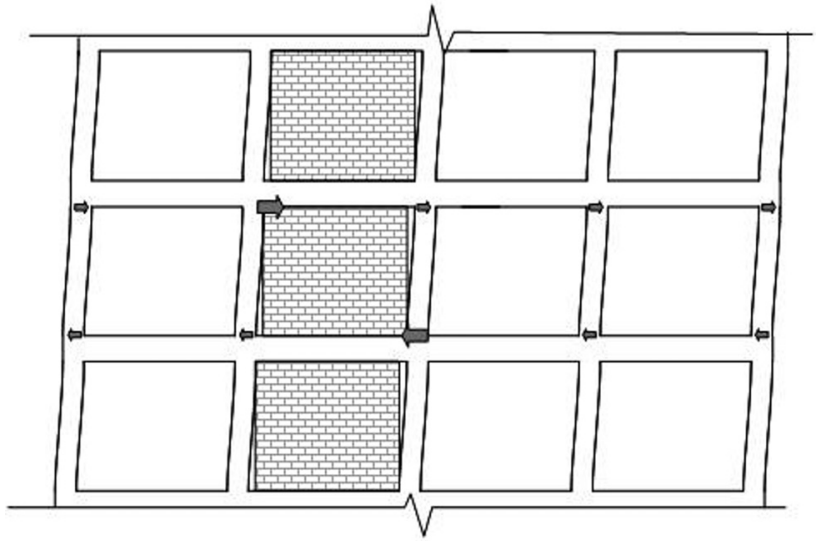

Figura 9

De entre los problemas de interacción entre la estructura y las fábricas inducidos por la degradación progresiva de éstas, que como ya se ha indicado se producía en las plantas inferiores, los que con mayor claridad se hacían patentes en Lorca eran:

1. Formación de mecanismos de tipo "planta blanda".

2. Daños en los pilares.

En lo que sigue se desarrollan ambos aspectos.

\section{Plantas blandas}

Para cuantificar este mecanismo de fallo se analiza la respuesta de un edificio convencional. Se considera representativo un bloque regular de 8 alturas cuya estructura, de hormigón armado, está construida por pórticos planos separados 6 $\mathrm{m}$ y formados por cuatro vanos con una anchura total de $20 \mathrm{~m}$. Todas las plantas tienen una altura de $3.0 \mathrm{~m}$ salvo la baja, en la que se supone $4.25 \mathrm{~m}$. Se utiliza un modelo de pórtico a cortante en el que las masas se estiman conforme a las prácticas constructivas habituales. Los pilares se dimensionan frente a las cargas gravitatorias, como era frecuente en los años 70 . El período fundamental de la estructura así configurada ronda el segundo ( $0.98 \mathrm{~s})$.

$\mathrm{Al}$ añadir la rigidez de tan sólo un paño de cerramiento por planta, de $5 \mathrm{~m}$ de longitud, el período se reduce a la mitad $(0.43 \mathrm{~s})$. Ello sitúa el edificio en las zonas de máxima amplificación del espectro (Figura 10) y conduce a valores muy elevados del cortante en la base (Figura 11). 


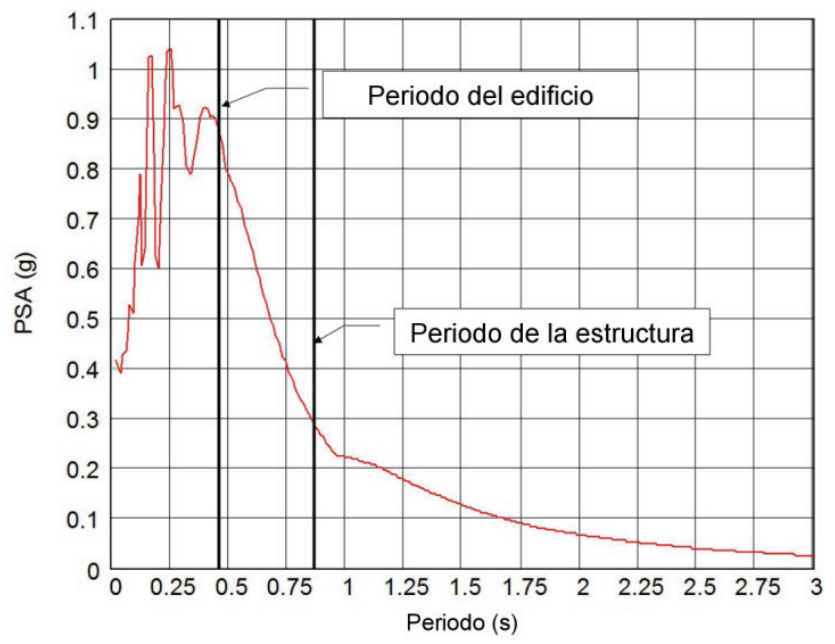

Figura 10

La mayor parte del citado cortante ha de soportarla la fábrica, conforme a su mayor rigidez. En concreto, el paño recoge las $2 / 3$ partes del cortante total, aproximadamente $2800 \mathrm{kN}$. Ello equivale a unas tensiones tangenciales medias que superan $\operatorname{los} 4 \mathrm{~N} / \mathrm{mm}^{2}$, valor muy superior a su resistencia.

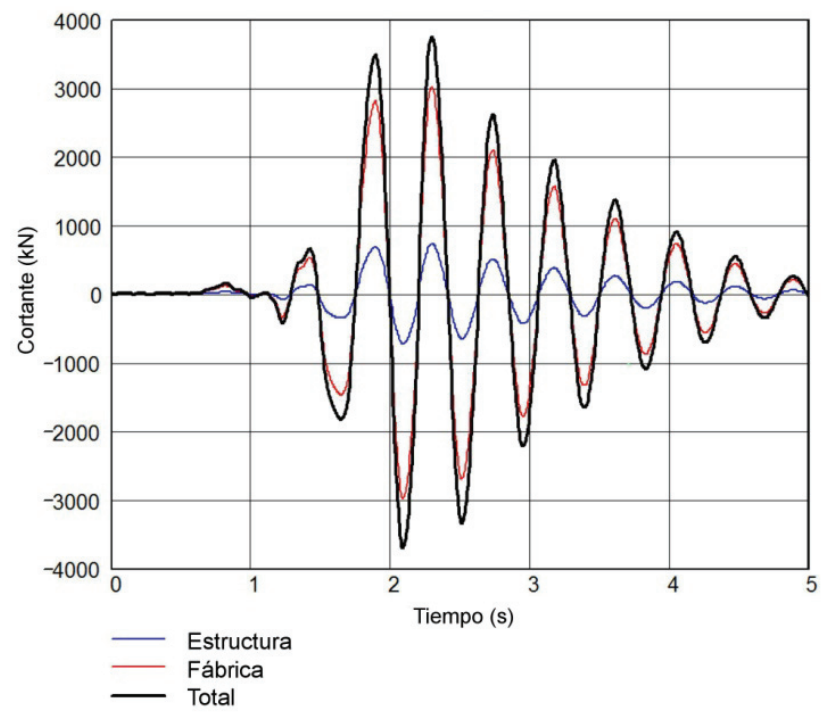

Figura 11

Lo anterior justifica la amplitud de los daños observados en los cerramientos y particiones (Figura 12). 


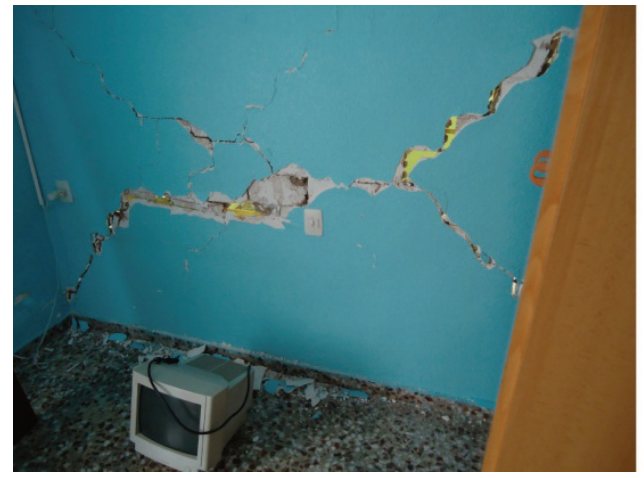

a)

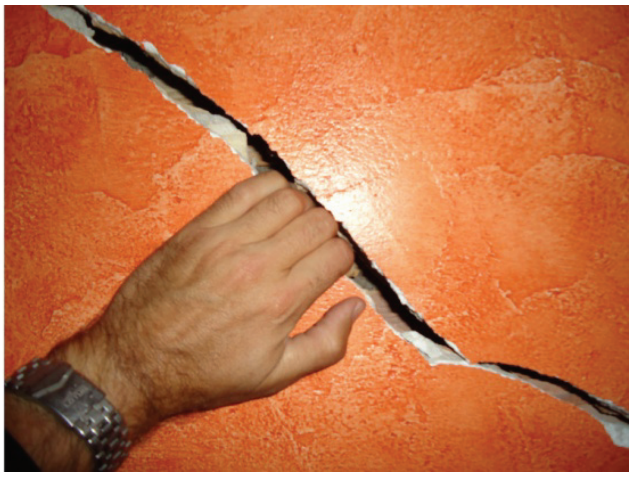

b)

Figura 12

En definitiva, mientras mantiene su integridad la fábrica aumenta el valor de los esfuerzos horizontales, lo que introduce solicitaciones muy importantes sobre la propia fábrica. Dichas solicitaciones producen, en la práctica generalidad de los casos observados, la rotura del paño.

Eliminando en el modelo la rigidez correspondiente al paño en la planta baja se obtiene un valor para el período intermedio $(0.68 \mathrm{~s})$ entre los ya citados. Igualmente, el cortante en la base adopta un valor intermedio, $2460 \mathrm{kN}$. Más importante que los valores concretos es el efecto de planta blanda que muestra la deformada modal. Así, en la figura 13a se muestra la forma correspondiente al edificio con un paño de fábrica en todas las alturas y en la (b)

Figura 13 b la del edificio en el que se ha eliminado el paño en planta baja.
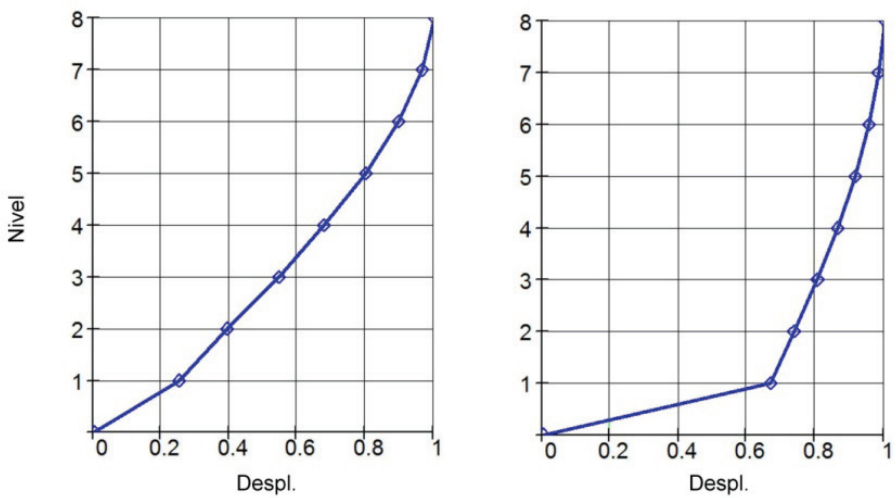

(a)

(b)

Figura 13 
En coherencia con la deformada que muestra la figura 13b la abertura de las fisuras en la fábrica alcanza valores (centímetros) muy superiores a los que se derivan del modelo rígido (un orden de magnitud inferiores).

Consecuencia igualmente de este efecto de planta blanda es la frecuencia con la que se observan impactos entre edificios aledaños y, especialmente, el que tales impactos se aprecien en los niveles inferiores (Figura 14).

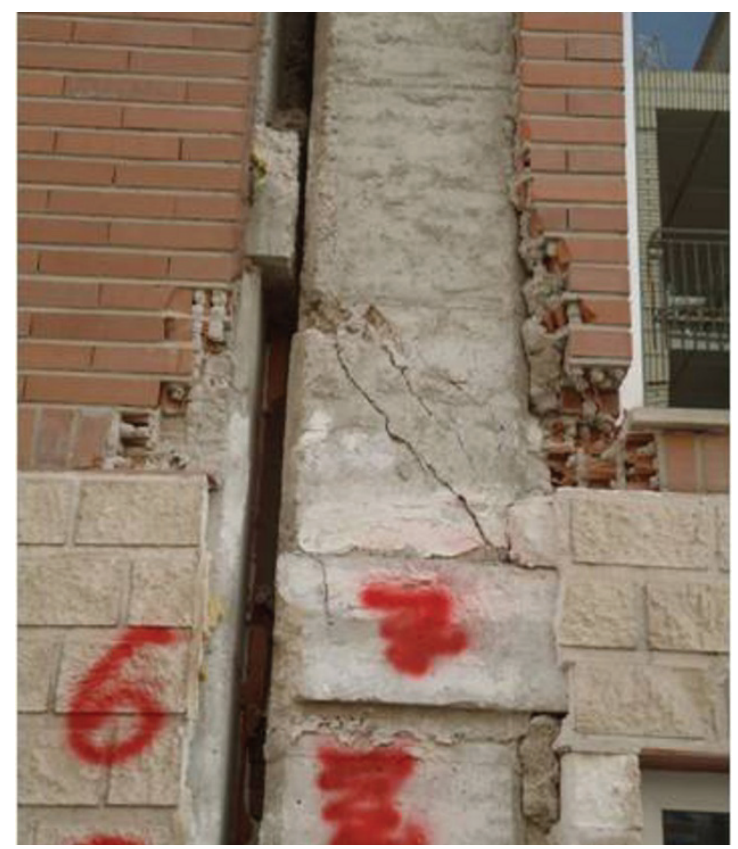

Figura 14

\section{Daños en pilares}

La interacción entre la fábrica y la estructura induce solicitaciones de gran valor sobre esta última, solicitaciones para las que no ha sido proyectada y que resultan especialmente graves por actuar sobre los elementos más importantes de la cadena resistente, los pilares.

La forma de rotura del paño determina las acciones sobre el pilar conforme a un planteamiento en capacidad: la solicitación sobre el pilar coincide con la resistencia de la fábrica en cada modo de fallo.

En muchas ocasiones la rotura de la fábrica se produce para cortantes bajos que no ponen en peligro el marco estructural. Ello suele ir acompañado de derrumbes que suponen un peligro para las personas. 
En otras ocasiones, sin embargo, se observa el fallo conjunto de la fábrica y el marco. Incluso, se han podido comprobar fallos de pilares previos al de la fábrica, que mantiene su integridad. Más normal es que sea el fallo de la fábrica el que condiciona la forma de fallo, posterior, del pilar. En cualquiera de ambos casos es la resistencia del conjunto paño de fábrica-marco estructural el que limita el cortante transmitido y condiciona, por tanto, la respuesta global del edificio. (Moehle et al. 2008).

En los criterios de proyecto actuales (EN1998-1, 2004) y en libros de texto para el diseño sísmico de hormigón armado y edificios de mampostería [0] indican la necesidad de tener en cuenta estos efectos.

Salvo en el caso de los pilares cortos, la mayoría de los fallos observados se producen en las plantas bajas de los edificios y responden a un proceso de interacción con la fábrica. Ello es coherente con los resultados de las comprobaciones más elementales que demuestran que para las alturas más habituales el fallo por flexión es siempre previo al fallo por cortante.

Supóngase, a modo de ejemplo, un pilar de sección cuadrada, de $40 \mathrm{~cm}$ de lado, construido en hormigón de $25 \mathrm{MPa}$ de resistencia característica y armado con tres redondos de $16 \mathrm{~mm}$ en cada cara. Si sobre el pilar actúa un axil equivalente a una tensión normal de $5 \mathrm{MPa}$ el agotamiento a flexión (Figura 15) se producirá para un momento de $215 \mathrm{kN} \cdot \mathrm{m}$.

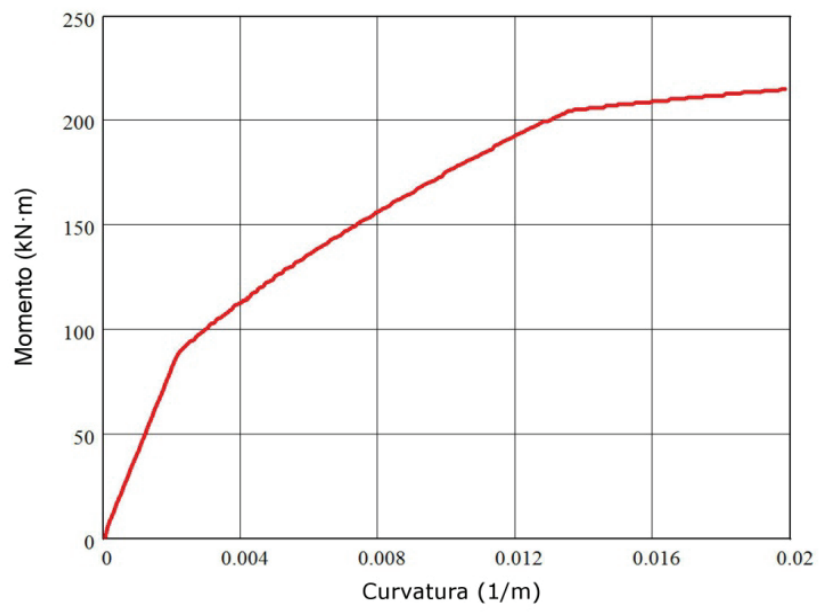

Figura 15

Un cálculo elemental conforme a los planteamientos de capacidad (Figura 16) permite obtener el valor máximo que puede llegar a pasar por el pilar, que suponiendo una altura de $4 \mathrm{~m}$ sería $107.5 \mathrm{kN}$, valor muy inferior a su resistencia a cortante. 


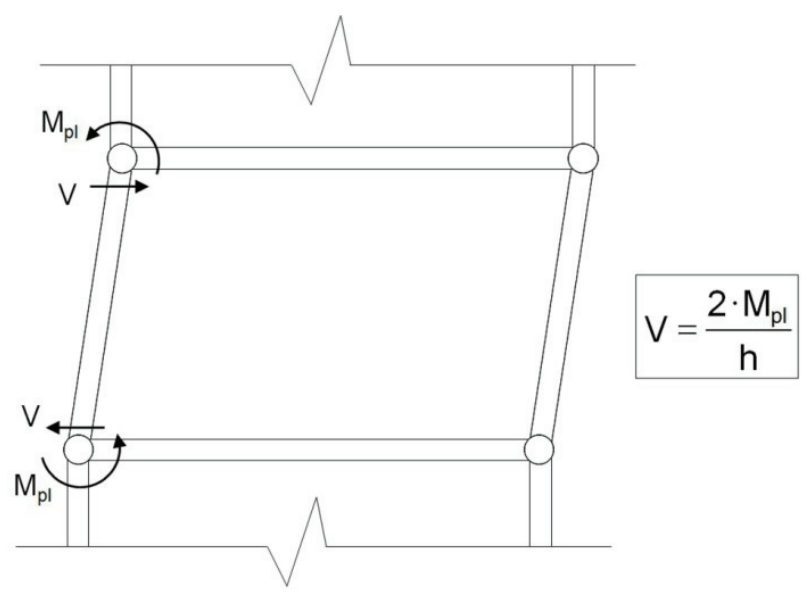

Figura 16

Efectivamente, conforme a la expresión de la norma Española (art. 44 de la EHE-08, 2008), semejante al Eurocódigo 2, para piezas sin armadura de cortante (hipótesis coherente con el deficiente estribado que se observaba en muchos casos):

$$
\mathrm{V}_{\mathrm{CU}}=\left[\frac{0.18}{\gamma_{c}} \cdot \xi \cdot\left(100 \cdot \rho_{1} \cdot \mathrm{f}_{\mathrm{cv}}\right)^{1 / 3}+0.15 \cdot \sigma_{\mathrm{cd}}^{\prime}\right] \cdot \mathrm{b}_{0} \cdot \mathrm{d}
$$

Se obtiene un valor de la resistencia al cortante en torno a $182.5 \mathrm{kN}$.

El mismo planteamiento en capacidad ya citado permite calcular de forma inmediata la altura mínima de un pilar que, con la sección y materiales considerados, fallase a corte. Sería:

$$
\mathrm{h}=\frac{2 \cdot \mathrm{M}_{\mathrm{pl}}}{\mathrm{V}_{\mathrm{R}}}=\frac{2 \cdot 215}{182.5}=2.36 \mathrm{~cm}
$$

Valor muy inferior al permitido por las normativas urbanísticas locales.

En definitiva, se comprueba la imposibilidad práctica del fallo a cortante de un pilar exento de las dimensiones habituales.

El presente artículo se centra precisamente en el análisis de esta interacción local entre la fábrica y la estructura. Se trataría, en primer lugar, de identificar las formas de fallo observadas en Lorca para, a continuación, comparar las resistencias de cada parte, fábrica y pilar. 


\section{Formas de fallo}

\subsection{Deslizamiento de la junta constructiva pilar-viga}

La junta de hormigonado entre vigas y pilares (Figura 17) puede suponer el eslabón más débil de la cadena resistente, especialmente si su ejecución no ha sido especialmente cuidada.

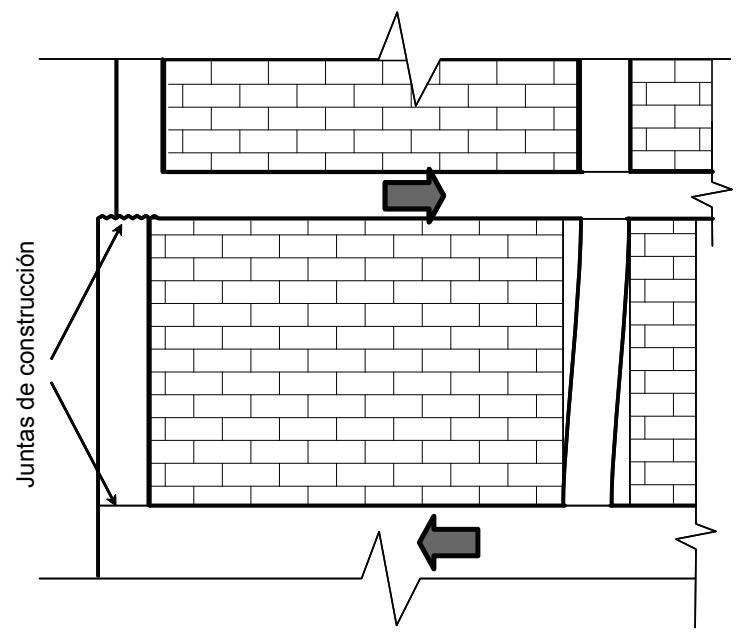

Figura 17

En Lorca se identificó este tipo de fallo en varios casos, localizados mayoritariamente en vanos extremos de fachada (Figura 18).

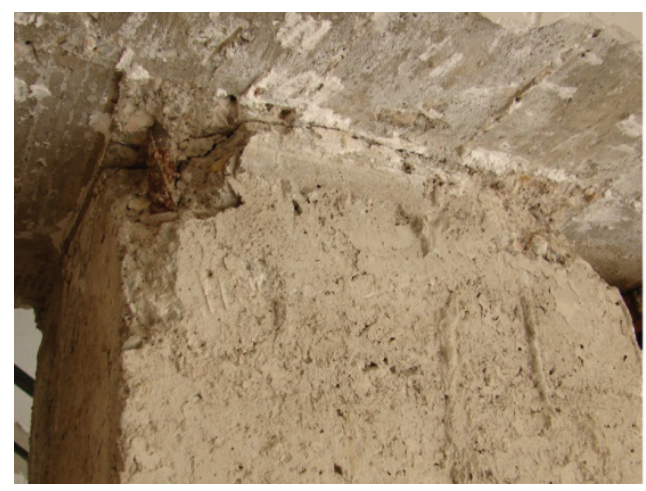

a)

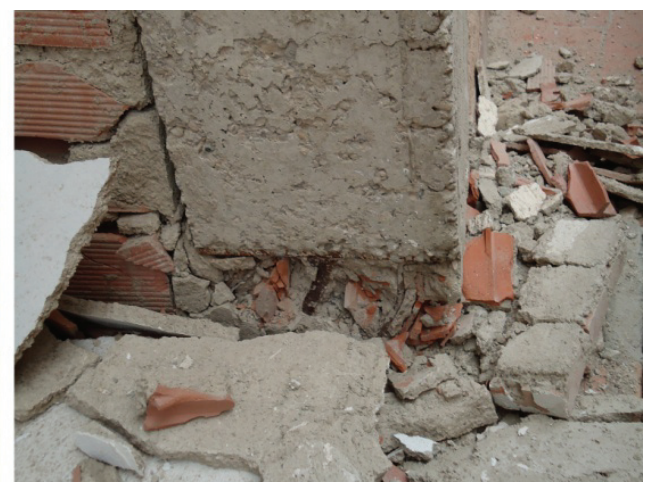

b)

Figura 18 


\subsection{Fallo del panel en tendeles}

Resulta especialmente peligroso porque sustituye la biela que forma el paño de fábrica (Figura 19 a)) por dos bielas de mayor inclinación (Figura 19 b)) que actúan directamente sobre las secciones intermedias del pilar.

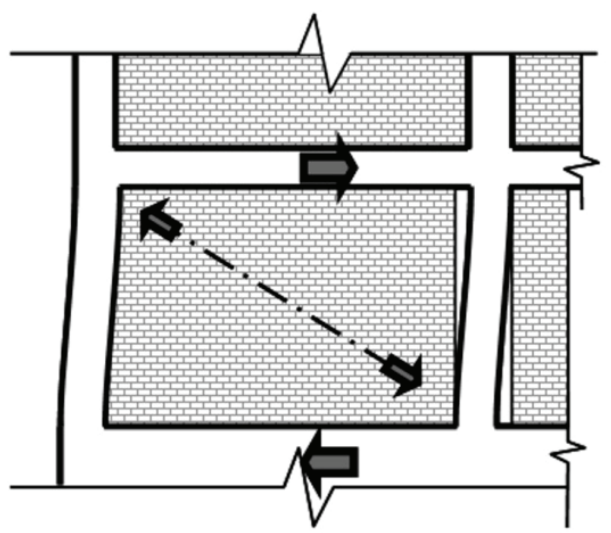

a)

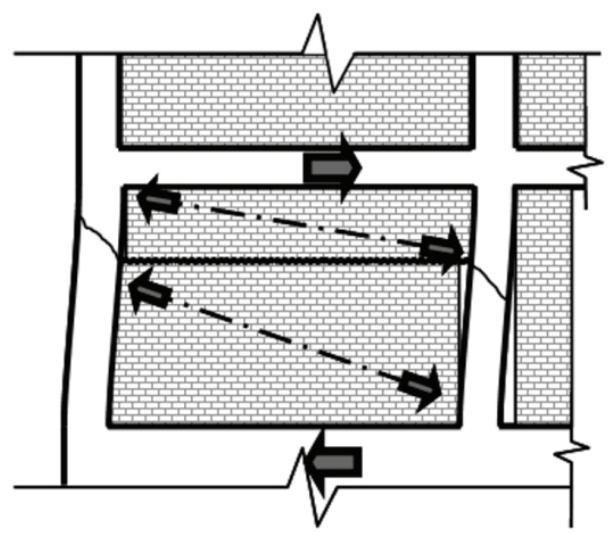

b)

Figura 19

En Lorca se ha observado este tipo de fallos asociados a discontinuidades del paño: huecos, rozas para conducciones, etc. (Figura 20).

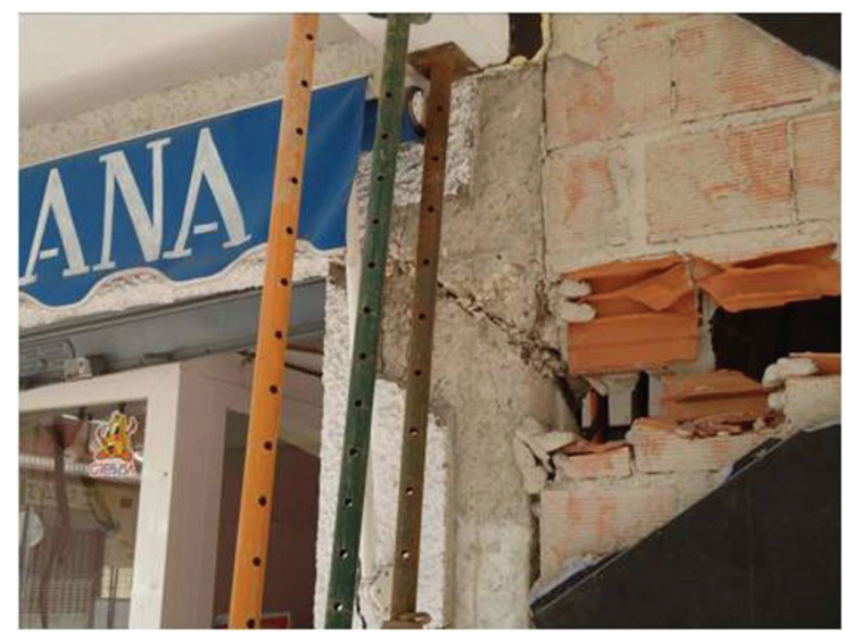

Figura 20 


\subsection{Fallo conjunto panel-pilar}

La fisuración habitual de la fábrica, marcando las diagonales traccionadas que se forman entre los nudos de esquina, se traslada en horizontal de tal forma que ambas diagonales, y las fisuras correspondientes, se cruzan en el centro de los pilares (Figura 21).

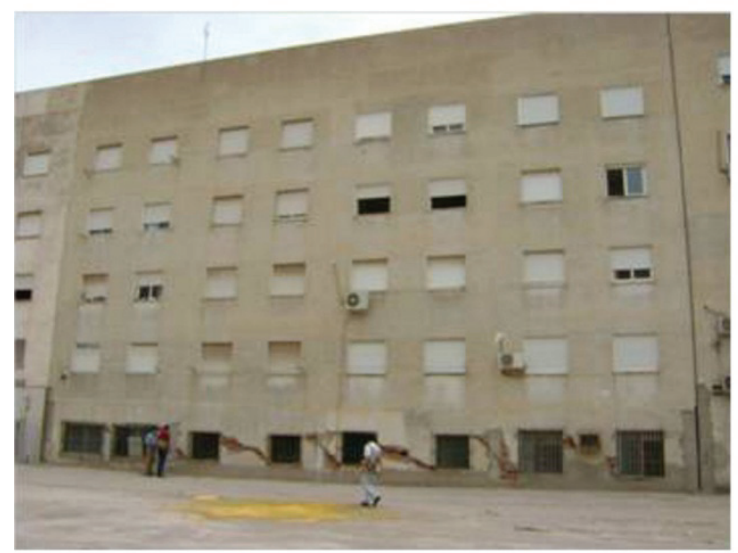

a)

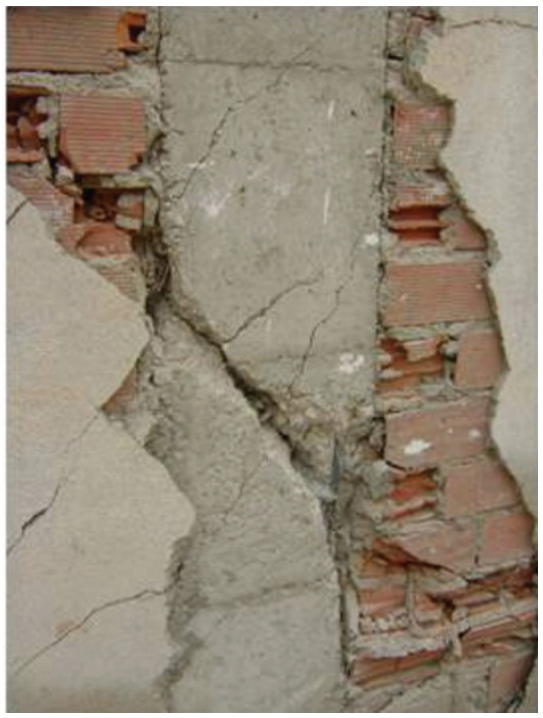

b)

Figura 21

\section{Solicitaciones sobre los pilares}

Con objeto de evaluar el nivel de solicitación que alcanzan los pilares antes de que la fábrica alcance el estado de fisuración, se ha procedido a realizar dos modelos de elementos finitos con mayor grado de detalle.

El primero es el edificio convencional indicado en el apartado 4, se representa por un pórtico interior en el que se contemplan 8 plantas la inferior de $4.25 \mathrm{~m}$ de altura y el resto de $3.00 \mathrm{~m}$ y cuatro vanos de $20 \mathrm{~m}$ de longitud total, donde en uno de los vanos interiores se introduce la fábrica en todas las alturas (Figura 22). 

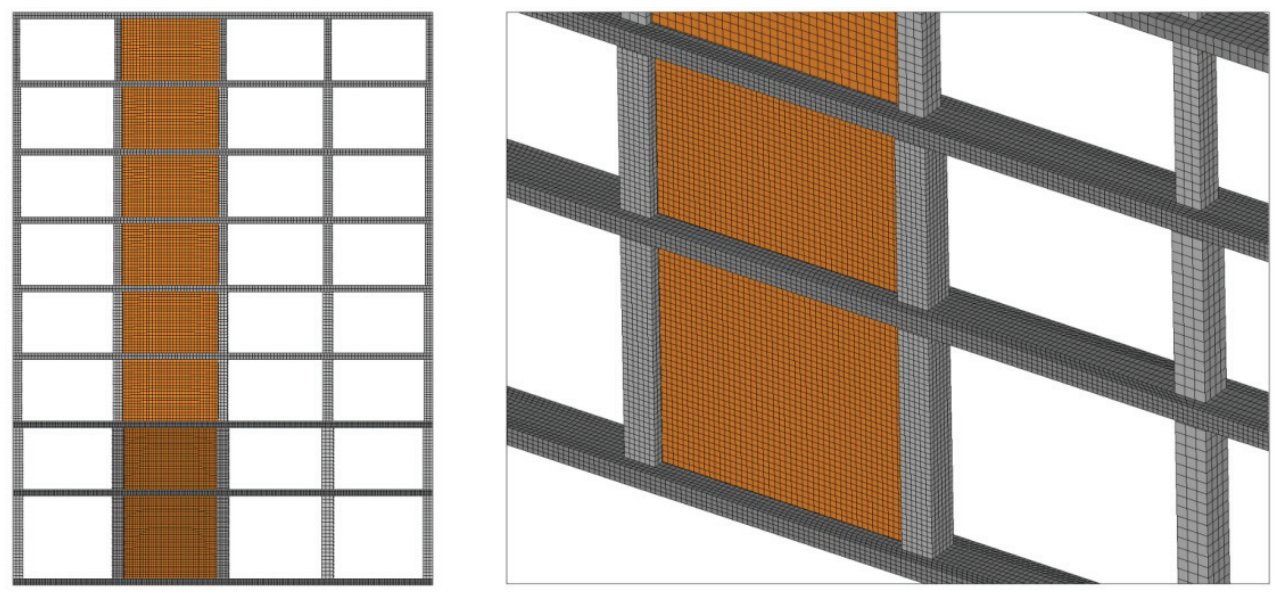

Figura 22

El dimensionamiento de la estructura conduce a pilares de tamaño variable en función de la altura, también distinguiendo entre los interiores y exteriores del pórtico según la distribución de la siguiente tabla.

Tabla 1

\begin{tabular}{|l|l|c|c|c|c|c|c|c|c|}
\hline $\begin{array}{c}\text { Sección } \\
\text { pilares }\end{array}$ & & nivel 1 & nivel 2 & nivel 3 & nivel 4 & nivel 5 & nivel 6 & nivel 7 & nivel 8 \\
\hline pilar & $\mathrm{m}^{2}$ & $\begin{array}{c}0.45 \mathrm{x} \\
\text { exterior }\end{array}$ & $\begin{array}{c}0.45 \mathrm{x} \\
\mathrm{n}\end{array}$ & $\begin{array}{c}0.40 \mathrm{x} \\
0.45\end{array}$ & $\begin{array}{c}0.40 \mathrm{x} \\
0.40\end{array}$ & $\begin{array}{c}0.35 \mathrm{x} \\
0.40\end{array}$ & $\begin{array}{c}0.35 \mathrm{x} \\
0.35\end{array}$ & $\begin{array}{c}0.35 \mathrm{x} \\
0.35\end{array}$ & $\begin{array}{c}0.30 \times \\
0.30\end{array}$ \\
\hline pilar & $\mathrm{m}^{2}$ & $\begin{array}{c}0.55 \mathrm{x} \\
\text { interior }\end{array}$ & $\begin{array}{c}0.55 \mathrm{x} \\
0.55\end{array}$ & $\begin{array}{c}0.45 \mathrm{x} \\
0.55\end{array}$ & $\begin{array}{c}0.45 \mathrm{x} \\
0.45\end{array}$ & $\begin{array}{c}0.40 \mathrm{x} \\
0.45\end{array}$ & $\begin{array}{c}0.40 \mathrm{x} \\
0.40\end{array}$ & $\begin{array}{c}0.40 \mathrm{x} \\
0.40\end{array}$ & $\begin{array}{c}0.30 \mathrm{x} \\
0.30\end{array}$ \\
\hline
\end{tabular}

Se emplean vigas planas como es habitual en edificación de la misma sección en todas las alturas $0.3 \times 1 \mathrm{~m}^{2}$. A las vigas se añade la masa tributaria de los materiales del forjado junto con la sobrecarga de uso como masa añadida.

El segundo corresponde a un edifico también de 8 plantas, en el que las alturas son de $4 \mathrm{~m}$ en planta baja y $3 \mathrm{~m}$ en el resto, con el mismo vano horizontal y la fábrica colocada en uno del los vanos exteriores también en todas las alturas (Figura 23). 

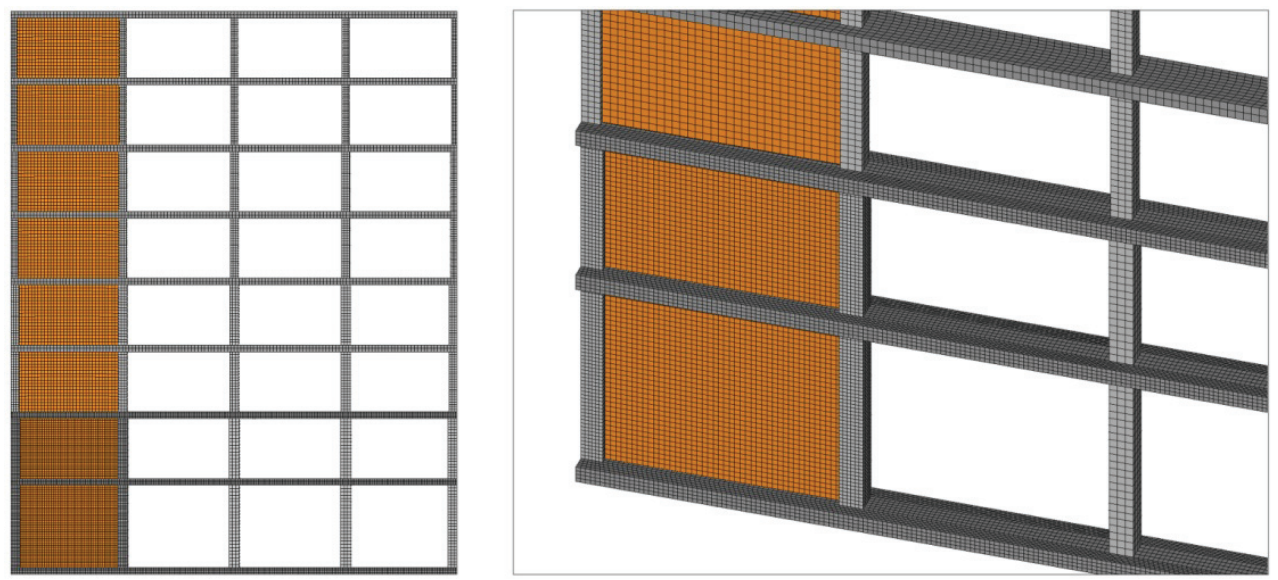

Figura 23

El dimensionamiento de los pilares en altura sigue la distribución indicada en la siguiente tabla, y se han mantenido las mismas características de las vigas:

Tabla 2

\begin{tabular}{|l|l|c|c|c|c|c|c|c|c|}
\hline $\begin{array}{c}\text { Sección } \\
\text { pilares }\end{array}$ & & nivel 1 & nivel 2 & nivel 3 & nivel 4 & nivel 5 & nivel 6 & nivel 7 & nivel 8 \\
\hline pilar & \multirow{2}{*}{$\mathrm{m}^{2}$} & $\begin{array}{c}0.40 \mathrm{x} \\
0.40\end{array}$ & $\begin{array}{c}0.40 \mathrm{x} \\
0.40\end{array}$ & $\begin{array}{c}0.35 \mathrm{x} \\
0.35\end{array}$ & $\begin{array}{c}0.35 \mathrm{x} \\
0.35\end{array}$ & $\begin{array}{c}0.30 \mathrm{x} \\
0.30\end{array}$ & $\begin{array}{c}0.30 \mathrm{x} \\
0.30\end{array}$ & $\begin{array}{c}0.25 \mathrm{x} \\
0.25\end{array}$ & $\begin{array}{c}0.25 \mathrm{x} \\
0.25\end{array}$ \\
\hline exterior & & $0.45 \mathrm{x}$ & $0.45 \mathrm{x}$ & $0.40 \mathrm{x}$ & $0.40 \mathrm{x}$ & $0.35 \mathrm{x}$ & $0.35 \mathrm{x}$ & $0.30 \mathrm{x}$ & $0.30 \mathrm{x}$ \\
pilar & \multirow{2}{*}{$\mathrm{m}^{2}$} & $\begin{array}{c}0.45 \\
\text { interior }\end{array}$ & 0.45 & 0.40 & 0.40 & 0.35 & 0.35 & 0.30 & 0.30 \\
\hline
\end{tabular}

Esta tipología corresponde a los edificios de los años 70 en contraposición al ejemplo anterior típico de construcciones actuales. Se pretende observar también la influencia de la colocación de la fábrica en el vano lateral.

En los modelos numéricos pilares y vigas se representan con elementos sólidos de 8 nudos elásticos con módulo de Young $2.7 \cdot 10^{10} \mathrm{~N} / \mathrm{m}^{2}$, coeficiente de Poisson 0.25 y la correspondiente densidad del material de $2500 \mathrm{~kg} / \mathrm{m}^{3}$.

La fábrica se coloca rellenando completamente el hueco entre vigas y pilares en cada planta con un espesor de $15 \mathrm{~cm}$ igual en todas las plantas. Se emplean también elementos sólidos de 8 nudos con módulo de Young $4 \cdot 10^{9} \mathrm{~N} / \mathrm{m}^{2}$, coeficiente de Poisson 0.3 y densidad $1600 \mathrm{~kg} / \mathrm{m}^{3}$. En estos elementos se introduce la capacidad de fisuración que se produce al alcanzar una tensión tangencial de $4 \cdot 10^{5}$ $\mathrm{N} / \mathrm{m}^{2}$.

La conexión entre la fábrica y el hormigón de vigas y pilares se realiza a través de elementos de contacto infinitamente rígidos en la dirección normal a la superficie y con un rozamiento de Coulomb $(\mu=0.15)$ en la dirección tangente. 
El análisis se realiza mediante un empuje progresivo (pushover) con condiciones de empotramiento en el nivel 0 . En el instante inicial se introduce el efecto de la gravedad que solicita la estructura y pre-comprime los paneles. En los siguientes instantes se procede a aplicar un desplazamiento incremental impuesto en las vigas de cada altura.

Se controla para cada incremento de desplazamiento el valor del cortante en la cabeza de los cuatro pilares de planta baja, así como el cortante que transmite la fábrica por efecto de rozamiento hasta el momento en el que se produce la fisuración. De la misma forma se solicitan el reparto del cortante a una distancia igual al canto del pilar por debajo de la junta viga-pilar.

En el primero de los modelos la fisuración del tabique en planta baja se produce cuando el desplazamiento relativo de la primera planta alcanza un valor de 5.3 $\mathrm{mm}$ (Figura 24).
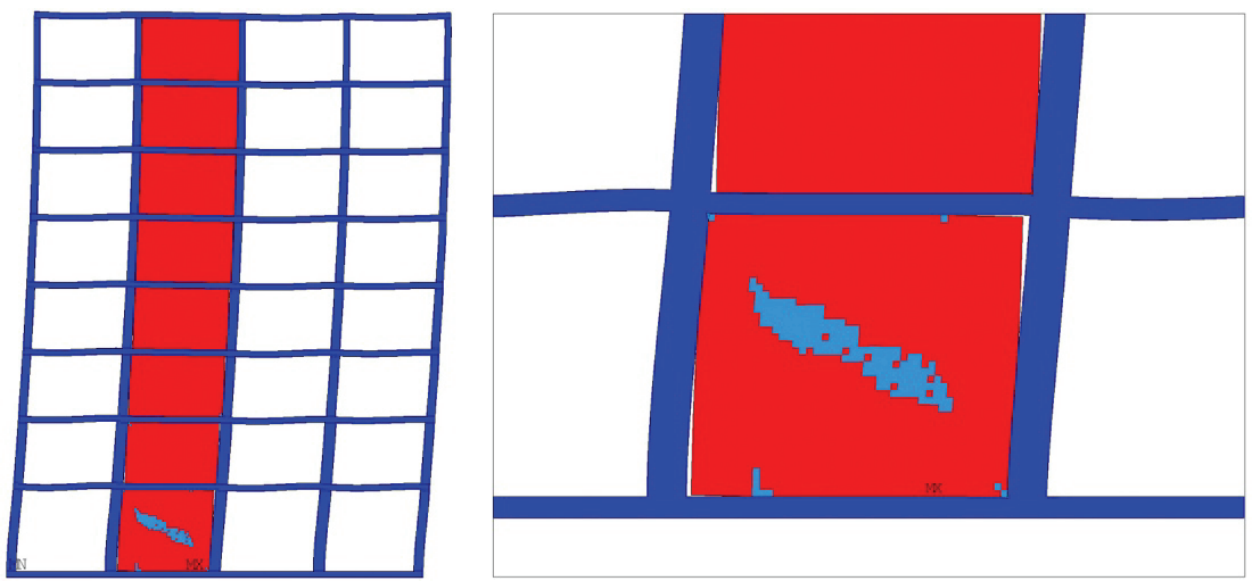

Figura 24

En la Figura 25 se representan las presiones que ejerce el tabique sobre el pilar en este instante, la fábrica actúa según se ha descrito en capítulos anteriores, la biela de compresión empuja al pilar aumentando de manera notable el cortante que tiene que transmitir. 


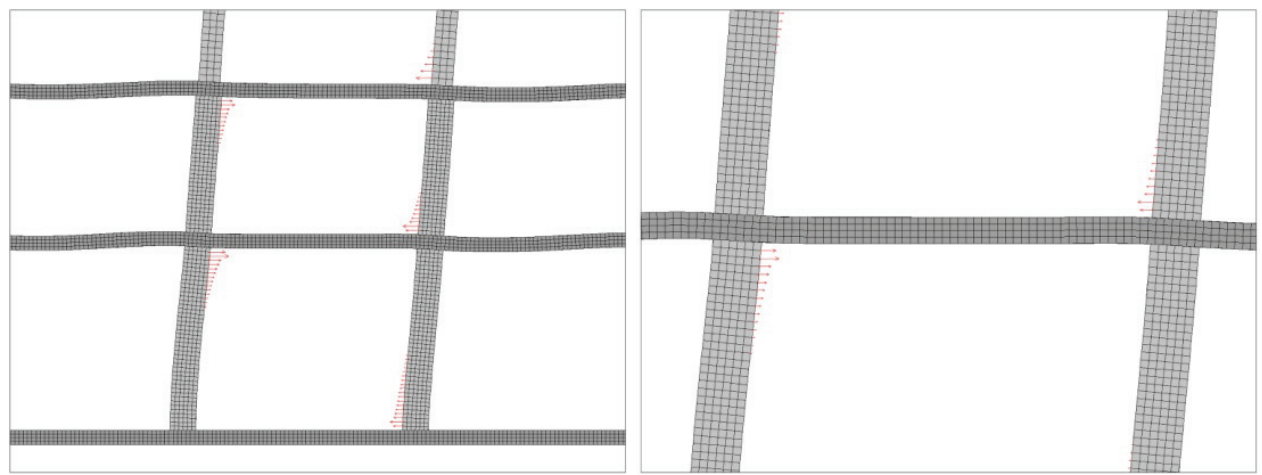

Figura 25

Los valores calculados en la simulación se muestran en el gráfico y tabla de la Figura 26, donde se puede observar el reparto del cortante en la cabeza de los pilares y por el tabique debido al rozamiento con la viga superior.

Según se observa el pilar más solicitado por la interacción con el tabique recoge el $62.2 \%$ del cortante total transmitido, con un valor de $569.2 \mathrm{kN}$, lo que se puede comparar con el nivel de la pila 4 (10.8\%) de idénticas dimensiones y sin fábrica en los vanos adyacentes. Por su parte, la fábrica recoge el $10.5 \%$ del cortante total al incluir las condiciones de rozamiento indicadas.

Cuando se analizan los niveles un canto por debajo de las anteriores se observa una reducción del cortante en el pilar hasta $189.9 \mathrm{kN}$ (aprox. 20\% del total). Siendo la fábrica la que transmite la diferencia del cortante en esa altura y transformándose en el elemento más cargado al asumir más del 50\% del cortante total.

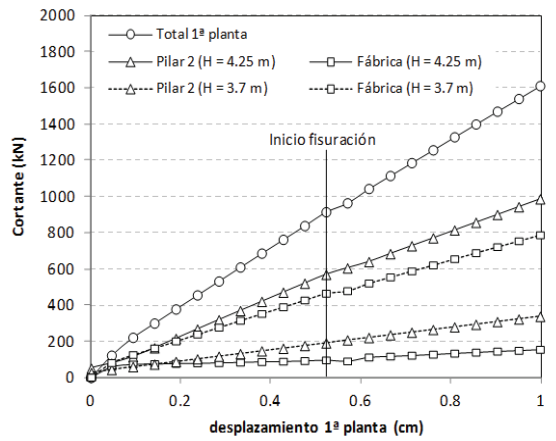

\begin{tabular}{|c|cc|cc|}
\cline { 2 - 5 } \multicolumn{1}{c|}{} & \multicolumn{2}{c|}{$\mathrm{H}=4.25 \mathrm{~m}$} & \multicolumn{2}{c|}{$\mathrm{H}=3.7 \mathrm{~m}$} \\
\cline { 2 - 5 } \multicolumn{1}{c|}{} & $\begin{array}{c}\text { Cortante } \\
\mathrm{kN}\end{array}$ & $\%$ & $\begin{array}{c}\text { Cortante } \\
\mathrm{kN}\end{array}$ & $\%$ \\
\hline Pilar 1 & 38.9 & $4.3 \%$ & 38.9 & $4.3 \%$ \\
Pilar 2 & 569.2 & $62.2 \%$ & 189.9 & $20.8 \%$ \\
Pilar 3 & 61.0 & $6.7 \%$ & 61.0 & $6.7 \%$ \\
Pilar 4 & 98.9 & $10.8 \%$ & 98.9 & $10.8 \%$ \\
Pilar 5 & 50.7 & $5.5 \%$ & 50.7 & $5.5 \%$ \\
Fábrica & 96.2 & $10.5 \%$ & 475.5 & $52.0 \%$ \\
\hline Total & 915.0 & $100.0 \%$ & 915.0 & $100.0 \%$ \\
\hline
\end{tabular}

Figura 26 
El mismo cálculo se ha repetido para la segunda estructura. Al ser más flexible la fisuración se alcanza con un valor del desplazamiento relativo de la primera planta de $6.3 \mathrm{~mm}$ (Figura 27), superior al ejemplo anterior.
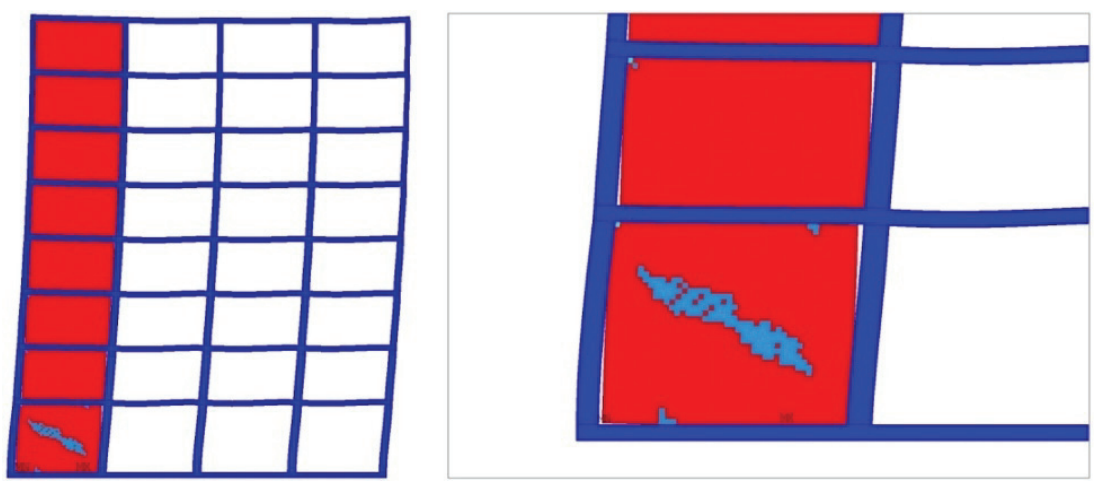

Figura 27

Sin embargo, como la condición impuesta en ambos casos se refiere al comienzo de la fisuración los valores alcanzados en el cortante en los puntos seleccionados no son muy diferentes (Figura 28). De manera porcentual el pilar más solicitado se lleva por encima del $60 \%$ del cortante total mientras que la fábrica transmite un $10 \%$ en su unión con la viga de primera planta.

Cuando se analizan estos mismos cortantes a una altura un canto inferior a la unión viga-pilar se observa que el pilar reduce el nivel hasta aproximadamente el $18 \%$ del total (pasa de $514 \mathrm{kN}$ hasta $149 \mathrm{kN}$ ) y la fábrica eleva su valor hasta algo más del $50 \%$. Todos estos valores son similares a los obtenidos en el primer ejemplo.

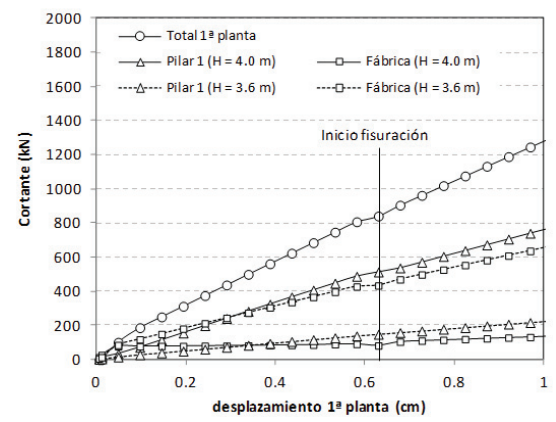

\begin{tabular}{|c|cc|cc|}
\cline { 2 - 5 } \multicolumn{1}{c|}{} & \multicolumn{2}{c|}{$\mathrm{H}=4.0 \mathrm{~m}$} & \multicolumn{2}{c|}{$\mathrm{H}=3.6 \mathrm{~m}$} \\
\cline { 2 - 5 } \multicolumn{1}{c|}{} & $\begin{array}{c}\text { Cortante } \\
\mathrm{kN}\end{array}$ & $\%$ & \multicolumn{2}{c|}{$\begin{array}{c}\text { Cortante } \\
\mathrm{kN}\end{array}$} \\
\hline Pilar 1 & 514.0 & $61.1 \%$ & 514.0 & $61.1 \%$ \\
Pilar 2 & 41.9 & $5.0 \%$ & 41.9 & $5.0 \%$ \\
Pilar 3 & 76.3 & $9.1 \%$ & 76.3 & $9.1 \%$ \\
Pilar 4 & 76.3 & $9.1 \%$ & 76.3 & $9.1 \%$ \\
Pilar 5 & 49.6 & $5.9 \%$ & 49.6 & $5.9 \%$ \\
Fábrica & 83.3 & $9.9 \%$ & 83.3 & $9.9 \%$ \\
\hline Total & 841.4 & $100.0 \%$ & 841.4 & $100.0 \%$ \\
\hline
\end{tabular}

Figura 28 


\section{Resistencia de los pilares}

Se analizan las dos formas de fallo del pilar descritas en los apartados precedentes, la correspondiente al deslizamiento de la junta constructiva entre los pilares y las vigas o losas de forjado (sección A-A' de la Figura 29) y la rotura convencional por agotamiento a tracción del alma, siguiendo el plano inclinado A-A" de la misma figura.

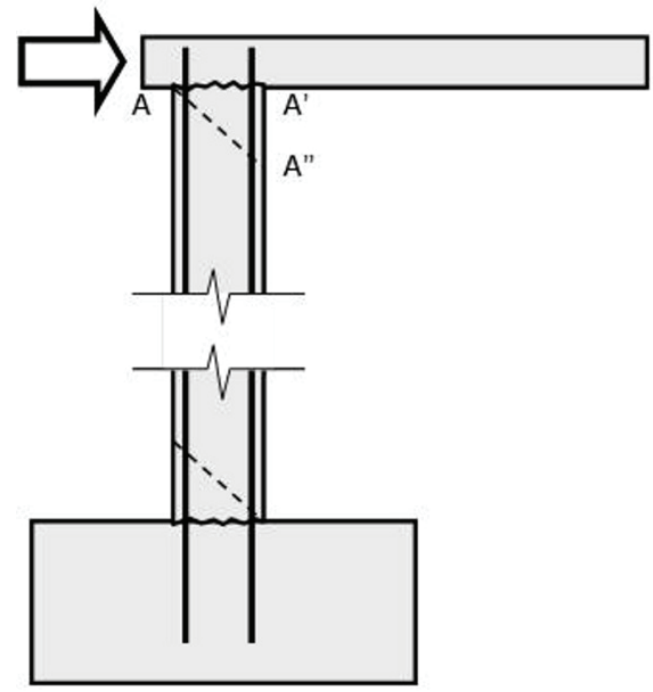

Figura 29

La resistencia al deslizamiento de la junta de construcción, sección A-A', viene dada en la norma Española (art. 47 de la EHE-08, 2008) por la expresión:

$$
\begin{aligned}
& \text { Si } \tau_{r, d} \leq 2.5 \beta\left(1.3-0.30 \frac{f_{c k}}{25}\right) f_{c t d}, y: \\
& \tau_{r, u}=\beta\left(1.3-0.30 \frac{f_{c k}}{25}\right) f_{c t, d}+\left(\frac{A_{s t}}{s p} f_{y \alpha, d}(\mu \operatorname{sen} \alpha+\cos \alpha)+\mu \sigma_{c d}\right) \leq 0.25 f_{c d}
\end{aligned}
$$

y en caso contrario:

$$
\tau_{r, u}=\left(\frac{A_{s t}}{s p} f_{y \alpha, d}(\mu \operatorname{sen} \alpha+\cos \alpha)+\mu \sigma_{c d}\right) \leq 0.25 f_{c d}
$$

Al comprobar la junta conforme a las expresiones anteriores se obtiene una resistencia, para la sección del pilar descrita en el apartado 4, de 769 kN. 
La resistencia a cortante por rotura a tracción del hormigón del alma cuando no se considera la armadura transversal ya ha sido calculada en el apartado $n^{\circ} 4$ precedente, obteniéndose un valor de $182.5 \mathrm{kN}$, esto es, aproximadamente la cuarta parte de la resistencia de la junta constructiva.

Al repetir los cálculos precedentes para distintas secciones y calidades de hormigón se obtienen resultados semejantes: En todos los casos la resistencia de la junta es muy superior a la del pilar. Las roturas observadas en Lorca y registradas también en algunos edificios de L'Áquila (Verderame et al. 2011) han de responder, por tanto, a causas ajenas al comportamiento de la estructura. Entre tales causas cabría citar:

1. Ejecución deficiente de la junta. No parece la causa más probable, toda vez que el parámetro determinante en la resistencia, la rugosidad de las superficies que la forman, no debería depender de la calidad de la ejecución.

2. Reducción del axil transmitido por el pilar. La realidad de muchos edificios construidos conforme a la práctica habitual en algunos países del Mediterráneo es que las cargas gravitatorias se canalizan en una muy importante proporción a través de los cerramientos y particiones, paños de fábrica muy potentes que se unen en todo su perímetro a la estructura portante. La fluencia de las vigas y la retracción de los pilares contribuyen a la progresiva transferencia de cargas gravitatorias desde la estructura a la fábrica, mucho más rígida.

Con la citada transferencia se pierde una de las componentes mas importantes de la resistencia a cortante, tanto de la junta como del propio pilar (la debida a las tensiones normales en la dirección de su eje), lo que implica la drástica reducción de los valores anteriormente calculados.

No obstante, cuando la resistencia a cortante del pilar por agotamiento a tracción del alma (es decir, la rotura convencional en el plano inclinado A-A" de la Figura 29) viene dada por la armadura transversal, podría ocurrir que la reducción del esfuerzo axil afectase de forma más significativa a la resistencia de la junta de construcción que a la del propio pilar, de forma que el fallo se produjese en la junta. En todo caso, se trata de una hipótesis poco creíble toda vez que los edificios en los que se ha observado este tipo de fallo fueron construidos en un momento (años 60 a 80 del pasado siglo) en el que no era normal dotar a los pilares de la armadura transversal necesaria para admitir su colaboración.

3. Concentración de las presiones en cabeza del pilar. Si las bielas que forma la fábrica concentran su empuje en la cabeza del pilar los valores del cortante a considerar en las comprobaciones de la junta son muy superiores a los que realmente solicitan al pilar en la formulación convencional (Figura 30). 


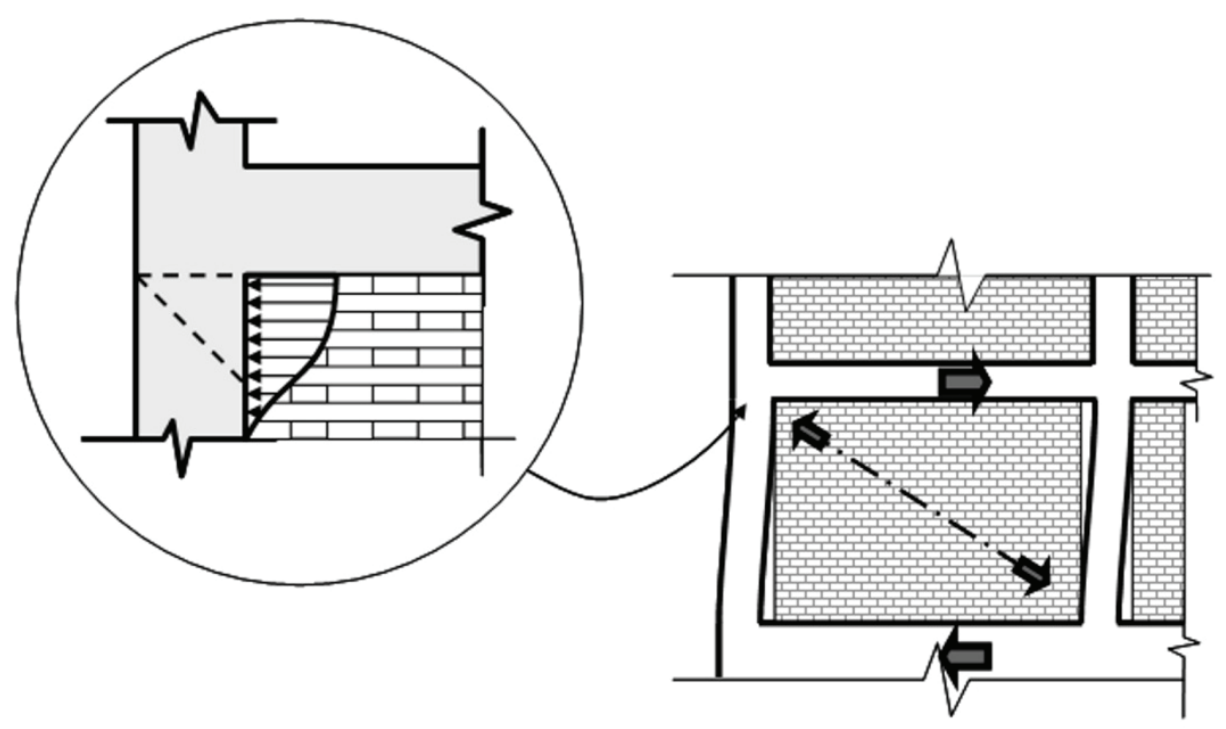

Figura 30

\section{Discusión de resultados}

Los resultados numéricos descritos en el apartado $\mathrm{n}^{\circ} 6$ justifican los daños en pilares a los que se hacía referencia más atrás. En efecto:

1. La existencia del panel de fábrica concentra en los pilares que lo confinan gran parte del cortante transmitido en la planta. En concreto, más de la mitad del cortante total actúa sobre uno sólo de los cinco pilares de la planta, frente al $10 \%$ que recoge el más cargado de los restantes. Esta concentración de esfuerzos es prácticamente independiente de la situación del panel (en un vano interior o exterior) y de la rigidez de los propios pilares, lo que es lógico porque la situación que se analiza, corresponde a la rotura de la fábrica. Estos valores estarían, por tanto, reflejando la resistencia de ésta.

2. Los paños de fábrica recogen una parte significativa de las cargas gravitatorias. En un análisis estático simplificado considerando únicamente las cargas gravitatorias, análisis en el que no se consideran los efectos de la fluencia y la retracción de la estructura de hormigón, la fábrica recoge ya una carga de valor comparable a la que recogen los pilares menos cargados. Naturalmente, esta proporción aumenta al considerar los citados efectos. Se trata de un aspecto de gran importancia porque gran parte de la resistencia a cortante de los pilares depende del axil que los solicita.

3. El análisis ante acciones horizontales demuestra que se pueden producir redistribuciones importantes de las cargas gravitatorias previas a la rotura de la fábrica, hasta el punto de llegar a cambiar el signo de los axiles en los pilares que enmarcan el paño. En definitiva, la fábrica actúa conforme 
al modelo convencional de biela comprimida, biela que introduce en el nudo superior una componente vertical de sentido ascendente y valor incluso superior a las cargas gravitatorias que descienden por el pilar (Figura 31). Conforme a la teoría clásica ello limitaría la resistencia a cortante de la junta entre pilar y viga a la capacidad, muy reducida, que puedan aportar las armaduras en un mecanismo de pasador. La capacidad del propio pilar a cortante se limitaría a la que pueda aportar la armadura transversal (aportación nula en los edificios anteriores a los años 80).

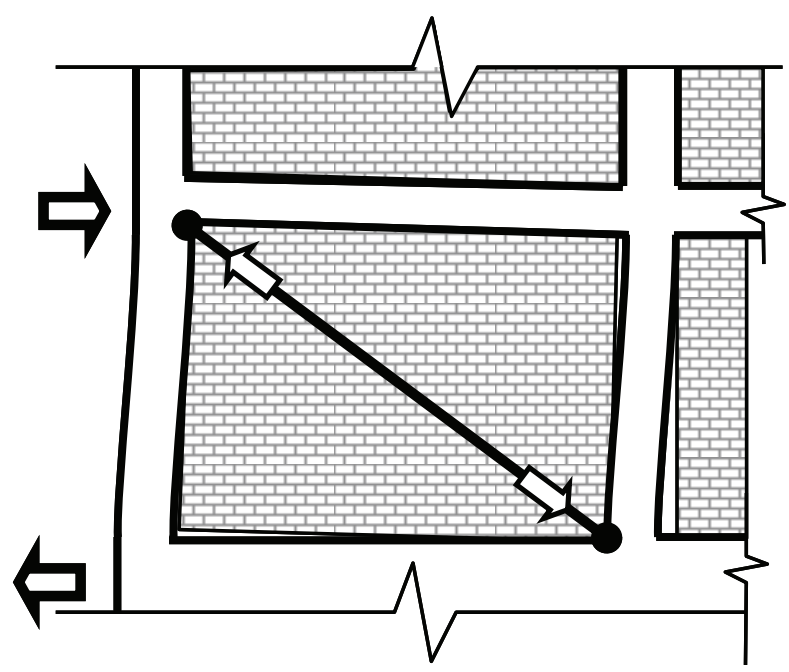

Figura 31

4. Es interesante señalar el paralelismo entre las resistencias asociadas a las dos formas de fallo, el del pilar y el de la junta con la viga, y las solicitaciones correspondientes a cada una.

5. Tabla 3). Cuando únicamente se consideran las cargas verticales y se omite el efecto de las fábricas, ambas resistencias son muy elevadas porque el axil que baja por el pilar en esta situación incrementa el término correspondiente al rozamiento. Si se tiene en cuenta, en cambio, la reducción del axil debido a la existencia de la fábrica ambas resistencias se reducen drásticamente. Se mantiene, no obstante, en paralelismo entre solicitación y resistencia a la que hacíamos referencia en el apartado precedente: Aunque la resistencia de la junta supere a la del pilar, las solicitaciones en ambos casos siguen una proporción similar: el pilar recibe una carga horizontal muy inferior a la que solicita la junta. En último 
término, si el pilar entra en tracción ambas resistencias se reducen hasta prácticamente anularse.

Tabla 3

\begin{tabular}{|c|c|c|c|}
\cline { 3 - 4 } \multicolumn{1}{c|}{} & \multirow{2}{*}{\multicolumn{1}{c|}{$\mathrm{V}_{\mathrm{S}}$}} & \multicolumn{2}{c|}{$\mathrm{V}_{\mathrm{R}}$} \\
\cline { 3 - 4 } \multicolumn{1}{c|}{} & & $\sigma=5 \mathrm{MPA}$ & $\sigma=2 \mathrm{MPA}$ \\
\hline JUNTA & $514 \mathrm{kN}$ & $769 \mathrm{kN}$ & $494 \mathrm{kN}$ \\
\hline PILAR & $149 \mathrm{kN}$ & $182.5 \mathrm{kN}$ & $119 \mathrm{kN}$ \\
\hline
\end{tabular}

\section{Referencias bibliográficas}

NCSE 2002 (2002). Norma de Construcción Sismoresistente: Parte general y edificación, Ministerio de Fomento, España (www.fomento.es)

EN1998-1 (2004). "Eurocode 8: Design of structures for earthquake resistance. Part 1 General rules, seismic actions and rules for buildings", European Committee for Standardization

CABAÑAS RODRÍGUEZ, L.; CARREÑO HERRERO, E.; IZQUIERDO ALVAREZ, A.; MARTÍNEZ SOLARES, J.M.; CAPOTE DEL VILLAR, R.; MARTÍNEZ DÍAZ, J.; BENITO OTERINO, B.; GASPAR ESCRIBANO, J.; RIVAS MEDINA, A.; GARCÍA MAYORDOMO, J.; PÉREZ LÓPEZ, R.; RODRÍGUEZ PASCUAL \& M.A.; MURPHY CORELLA, P. (2011) Informe del sismo de Lorca del 11 de mayo de 2011, Instituto Geográfico Nacional (www.ign.es).

FEMA E-74 (2011). Reducing the Risks of Nonstructural Earthquake Damage- A Practical Guide, Fourth Edition, FEMA (www.fema.gov)

MOEHLE, JACK P., HOOPER, JOHN D., AND LUBKE, CHRIS D. (2008) Seismic design of reinforced concrete special moment frames: a guide for practicing engineers, NEHRP Seismic Design Technical Brief No. 1

PAULAY T, PRIESTLEY MJN (1992) Seismic Design of Reinforced Concrete and Masonry Buildings. Wiley Interscience. ISBN: 0-471-54915-0

EHE (2008). Instrucción de Hormigón Estructural Ministerio de Fomento. NIPO: $161-11-150-2$

VERDERAME, G.M; LUCA, F; RICCI, G; MANFREDI, G. (2011). Preliminary analysis of a soft-storey mechanism after the 2009 L'Aquila earthquake. Earthquake Engineering \& Structural Dynamics. 40:8, 925-944 